\title{
Calpain-mediated cleavage of Beclin-1 and autophagy deregulation following retinal ischemic injury in vivo
}

\author{
R Russo*,1, L Berliocchi ${ }^{2}$, A Adornetto', GP Varano', F Cavaliere', C Nucci ${ }^{1}$, D Rotiroti ${ }^{2}$, LA Morrone ${ }^{1,4}$, G Bagetta ${ }^{1,4}$ \\ and MT Corasaniti ${ }^{2}$
}

Autophagy is the major intracellular degradation pathway that regulates long-lived proteins and organelles turnover. This process occurs at basal levels in all cells but it is rapidly upregulated in response to starvation and cellular stress. Although being recently implicated in neurodegeneration, it remains still unclear whether autophagy has a detrimental or protective role. In this study, we investigated the dynamics of the autophagic process in retinal tissue that has undergone transient ischemia, an experimental model that recapitulates features of ocular pathologies, including glaucoma, anterior ischemic optic neuropathy and retinal vessels occlusion. Retinal ischemia, induced in adult rats by increasing the intraocular pressure, was characterized by a reduction in the phosphatidylethanolamine-modified form of LC3 (LC3II) and by a significant decrease in Beclin-1. The latter event was associated with a proteolytic cleavage of Beclin-1, leading to the accumulation of a 50-kDa fragment. This event was prevented by intravitreal treatment with the non-competitive $\mathrm{N}$-methyl-D-aspartate antagonist MK801 and calpain inhibitors or by calpain knockdown. Blockade of autophagy by pharmacological inhibition or Beclin-1 silencing in RGC-5 increased cell death, suggesting a pro-survival role of the autophagic process in this neuronal cell type. Altogether, our results provide original evidence for calpain-mediated cleavage of Beclin-1 and deregulation of basal autophagy in the rat retina that has undergone ocular ischemia/reperfusion injury.

Cell Death and Disease (2011) 2, e144; doi:10.1038/cddis.2011.29; published online 14 April 2011

Subject Category: Neuroscience

Autophagy is an evolutionarily conserved process by which eukaryotic cells regulate the turnover of long-lived proteins and cytoplasmic organelles. Depending on mechanisms and functions, three types of autophagy have been described in mammalians: macroautophagy, microautophagy and chaperone-mediated autophagy. ${ }^{1}$

Macroautophagy (hereafter referred to as autophagy) is the most prevalent form and involves the formation of the autophagosome. This is a double-membrane structure that, following engulfment of cytoplasmic components such as proteins, lipid and damaged organelles, docks and fuses with the lysosomal compartment to form the autolysosome. Here, the content of the autophagosome is degraded by the lysosomal hydrolases and recycled for macromolecular synthesis and ATP generation.

Autophagy occurs at low basal levels in virtually all cells performing homeostatic function, and acts as a catabolic adaptive process in response to different forms of stress, including starvation, growth factor withdrawal, high bioenergetic demands, hypoxia, endoplasmic reticulum stress or accumulation of protein aggregates. ${ }^{2}$
Besides its participation in neuronal homeostasis, ${ }^{3}$ development and remodeling, ${ }^{4,5}$ autophagy has recently drawn increasing attention for its role in neuronal cell death associated with chronic neurodegenerative disorders and acute neuronal injury. ${ }^{6}$ Dysfunctions in the autophagic process have been linked to Alzheimer's disease (AD), Parkinson's disease (PD), Huntington's disease, as well as to brain hypoxia/ischemia or trauma. ${ }^{7}$

Retinal ischemia is a common clinical condition representing the main cause of visual impairment and blindness. ${ }^{8}$ Ischemic phenomena occur in a variety of ocular pathologies, including anterior ischemic optic neuropathy, retinal and choroidal vessel occlusion, diabetic retinopathy, traumatic optic neuropathy and glaucoma. ${ }^{8,9}$

Common hallmark for these conditions is the progressive degeneration and the final loss of the retinal ganglion cells (RGCs) that through the optic nerve fibers transmit the visual information to the central areas of the visual pathway. Block of blood flow to the retina triggers a self-reinforcing detrimental cascade involving neuronal depolarization, calcium overload, oxidative stress and alteration of glutamate homeostasis. ${ }^{10}$

\footnotetext{
'Department of Pharmacobiology, University of Calabria, Arcavacata di Rende, Italy; 'Department of Pharmacobiological Sciences, University 'Magna Graecia' of Catanzaro, Catanzaro, Italy; ${ }^{3}$ Physiopathological Optics, Department of Biopathology, University of Rome 'Tor Vergata', Rome, Italy and ${ }^{4}$ University Center for Adaptive Disorders and Headache, Section of Neuropharmacology of Normal and Pathological Neuronal Plasticity, University of Calabria, Arcavacata di Rende, Italy *Corresponding author: R Russo, Department of Pharmacobiology, University of Calabria, 87036 Arcavacata di Rende, Italy. Tel: + 390984493455 ; Fax: + 390984 493064; E-mail: rossella.russo@unical.it Keywords: autophagy; retinal ischemia; calpain; Beclin-1; LC3

Abbreviations: LC3II, phosphatidylethanolamine-modified form of LC3; RGCs, retinal ganglion cells; NMDA, N-methyl-D-aspartate; IOP, intraocular pressure; PI3K, phosphatidylinositol-3-kinase; GCL, ganglion cell layer; SBDP, $\alpha$-spectrin breakdown product; CAPNS1, calpain small-subunit 1; SD, serum deprivation; BafA1, bafilomycin A1; 3-MA, 3-methyladenine; IsiRNA, small interfering RNA; MTT, 3-(4,5-Dimethyl-2-thiazolyl)-2,5-diphenyl-2H-tetrazolium bromide; GFAP, glial fibrillary acidic protein

Received 01.2.11; revised 03.3.11; accepted 07.3.11; Edited by P Nicotera
} 
In turn, the disruption of glutamate homeostasis leads to excitotoxic neuronal damage through the overactivation of $\mathrm{N}$ methyl-D-aspartate (NMDA) and non-NMDA glutamate receptors. ${ }^{10,11}$ At cellular level, transient retinal ischemia results in extensive neuronal cell death characterized by an early phase of necrosis and a prolonged phase of apoptosis. ${ }^{12}$

Aim of this study was to investigate the dynamics of the autophagic process in the retina by using both an in vivo model of ocular ischemia induced by the transient elevation of the intraocular pressure (IOP) and RGCs exposed to serum withdrawal. Our results showed that autophagy deregulation occurs during retinal ischemia. This was associated with Beclin-1 cleavage mediated by calpains and dependent on NMDA receptor activation. Furthermore, Beclin-1 silencing reduced $R G C$ viability under starvation, thus suggesting a pro-survival role for autophagy in this experimental context.

\section{Results}

Beclin-1 localizes mainly in the ganglion cell layer of the intact retina. Beclin-1 is part of a class III phosphatidylinositol-3-kinase (PI3K) complex that participates in the early steps of the autophagic vesicles formation, and it is essential for the recruitment of other Atg proteins to the preautophagosomal structure. ${ }^{13}$

Beclin-1 distribution in the retina was investigated by immunofluorescence. In intact retinas, Beclin-1 immunoreactivity was diffused throughout all retina layers, with a higher expression in the inner segment and particularly in the ganglion cell layer (GCL) as shown in Figure 1a. To identify the type of cell expressing Beclin-1, double-labeling experiments with RGC and Müller cell markers were performed. In the GCL, Beclin-1 immunoreactivity partially colocalized with the cytosolic and dendritic compartments of TUJ1-labeled RGCs and with the glial fibrillary acidic protein (GFAP)positive Müller cell processes and end-feet-surrounding RGCs as shown in Figures $1 b$ and $c$.

Beclin-I and LC3II levels decrease under retinal ischemia-reperfusion. LC3I is a cytosolic protein whose lipidated form, LC3II (phosphatidylethanolamine-modified form of LC3), is stably associated to the autophagosomal membrane and is therefore considered an autophagyspecific marker. ${ }^{14}$ To determine whether an ischemic insult can modulate autophagy in the retina, we monitored the expression of LC3 and Beclin-1 by western blot in rat retinas that have undergone high IOP.

Compared with the contralateral, non-ischemic eye, LC3II levels were significantly reduced in the ischemic retina between 0 and $1 \mathrm{~h}$ of reperfusion (Figure $2 \mathrm{a}$ ) and returned to basal levels after $24 \mathrm{~h}$ of reperfusion; no significant changes of the LC3 cytosolic form (LC3I) were detected over the analyzed time points (Figure 2a).

The LC3II reduction was associated with a significant decrease of Beclin-1 in the post-ischemic phase. Following ischemia, Beclin-1 expression was below the constitutive level during the first $24 \mathrm{~h}$ of reperfusion, showing a more pronounced and significant decrease after $1 \mathrm{~h}$ of reperfusion (Figure 2b). In the retina subjected to ischemia, Beclin-1 was
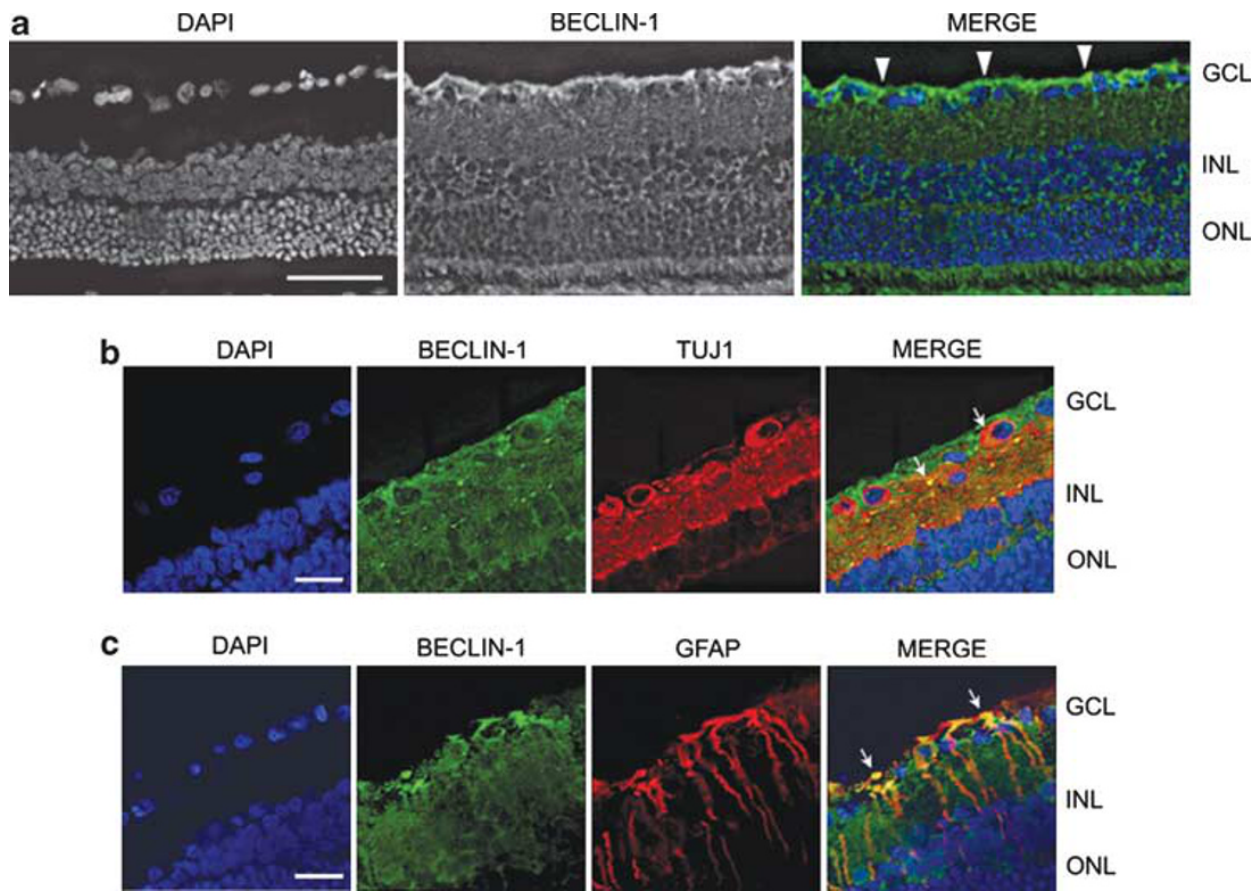

Figure 1 Expression pattern of Beclin-1 in the retina. (a) Representative tissue section of adult rat retina stained with primary antibody for Beclin-1. Immunofluorescence experiments show the diffused expression of Beclin-1 (green) in all retina layers and the more intense immunoreactivity in the GCL (white arrowheads). Cell nuclei were counterstained with DAPI. Scale bar $=50 \mu \mathrm{m}$. (b) Immunolocalization of Beclin-1 in RGCs. Beclin-1 immunoreactivity (green) was present in the cytoplasmatic and dendritic compartments of TUJ1-immunoreactive RGCs (red; white arrows). (c) Double fluorescence immunolabeling of Beclin-1 (green)/GFAP (red) shows colocalization (yellow signal; white arrows) in the Müller cell processes surrounding RGCs. Scale bar $=20 \mu \mathrm{m}$. INL, inner nuclear layer; ONL, outer nuclear layer 


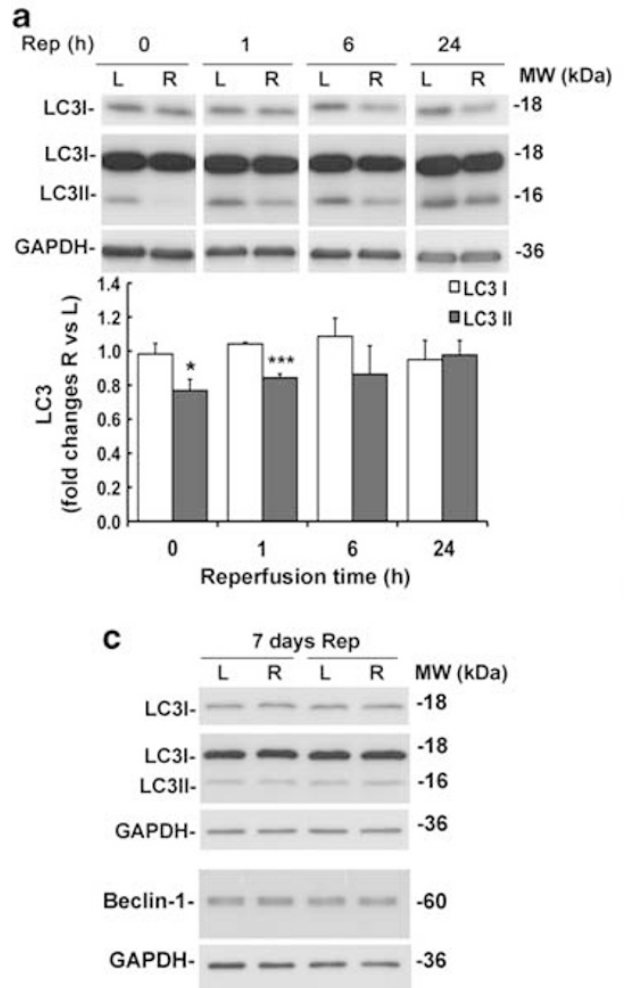

b
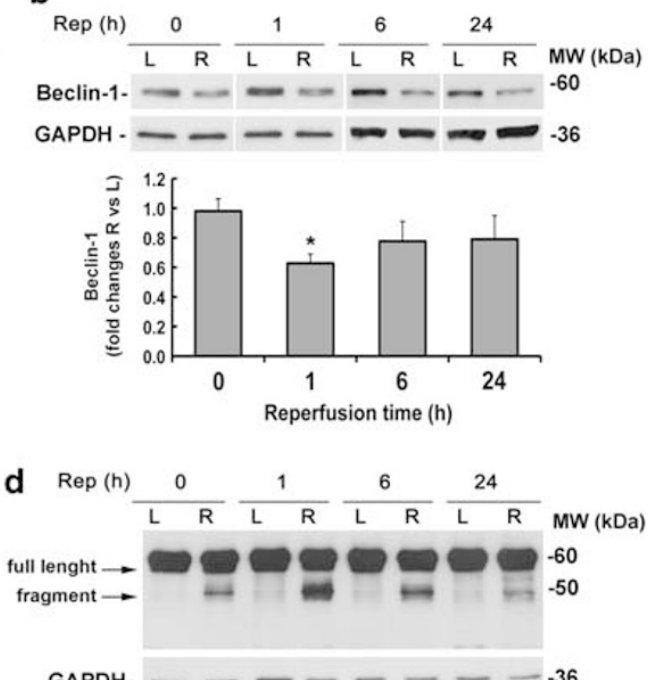

GAPDH. - - - - - - - -36

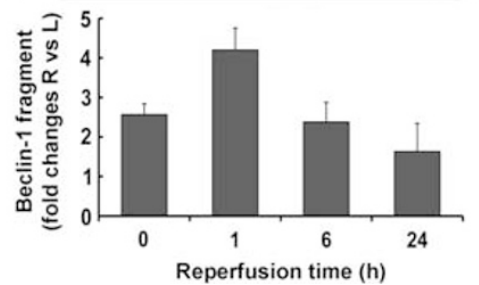

Figure 2 Time-dependent changes of LC3 and Beclin-1 following retinal ischemia are paralleled by Beclin-1 proteolytic cleavage. Rats were subjected to retinal ischemia in the right eye $(\mathrm{R})$ for $50 \mathrm{~min}$ and killed at the indicated time point (Rep, reperfusion time). For each animal, left eye $(\mathrm{L})$ was used as control. Total proteins were analyzed by western blotting for LC3 (a and $\mathbf{c}$ ) or Beclin-1 (b-d), and equal loading was confirmed by immunoblotting with anti-GAPDH antibody. Histograms show the result of the densitometric analysis after normalization using the internal loading control. (a) In the ischemic retina (R), LC3Il levels were significantly decreased at the end of the ischemia (Rep 0) and maintained below basal levels during the first hour of reperfusion. (b) Ischemia-induced Beclin-1 reduction with a peak at $1 \mathrm{~h}$ of reperfusion as compared with the left non-ischemic retina. (c) LC3 and Beclin-1 expression after 7 days of reperfusion. (d) Representative immunoblot showing the appearance and accumulation of the 50-kDa proteolytic fragment of Beclin-1 during the post-ischemic phase. Note that fragment was detectable only by longer exposure time compared with the full-length protein. Bars represent mean \pm S.E.M. from five to six rats in each group. ${ }^{*} P<0.05,{ }^{* \star *} P<0.001$ versus $L$. L, left retina; MW, molecular weight; $R$, right ischemic retina

reduced by $36 \%$ at $1 \mathrm{~h}$ of reperfusion when compared with the protein level in the non-ischemic retina (Figure $2 b$ ).

After 7 days from the ischemic insult, the ischemic retinas showed Beclin-1 and LC3II levels comparable to the basal levels of non-ischemic retinas (Figure 2c).

\section{Beclin-1 undergoes proteolytic cleavage following} retinal ischemia-reperfusion. In retinas that have undergone high IOP, Beclin-1 reduction was accompanied by the appearance of an additional band reactive to the antiBeclin-1 antibody and characterized by a molecular weight of $50 \mathrm{kDa}$ (Figure 2d). This fragment accumulated during the first hour of reperfusion and then slowly decreased over the $24 \mathrm{~h}$ (Figure 2d), paralleling Beclin-1 reduction and thus suggesting that cleavage of the full-length protein had occurred.

Retinal ischemia leads to calpain activation. Previous work from our and other groups showed the contribution of excitotoxicity to neurodegeneration induced by ischemia in the retina. ${ }^{11,15}$ Overactivation of glutamate receptors, mainly the NMDA subtypes, results in calcium overload and consequent activation of calcium-dependent enzymes. ${ }^{16}$ Together with the caspase cascade, also activation of the calcium-dependent cysteine proteases, calpains, occurs in excitotoxicity and represents an early step in the sequence of events leading to neuronal death.

To investigate activation of calpains in our model, we monitored the formation of $\alpha$-spectrin breakdown products (SBDPs) by western blot. In contrast to caspase-3, generating a $150-\mathrm{kDa}$ and an apoptotic-specific 120-kDa fragment, calpains cleave non-erythroid $\alpha$-spectrin into two breakdown products of 150 and $145 \mathrm{kDa}^{17}$

The appearance of the typical 150/145-kDa doublet was observed after $50 \mathrm{~min}$ of ischemia (reperfusion time 0 ), indicating a significant calpain activation (Figures $3 a$ and b). Calpain activation further increased during the first hour of reperfusion and was maintained at 6 and $24 \mathrm{~h}$ of reperfusion (Figures $3 a$ and $b$ ), showing a temporal profile overlapping the appearance of the Beclin-1 fragment (Figure 2d). No significant modulation of the caspase-3-mediated $120-\mathrm{kDa}$ SBDP was detected at the same time points (Figures $3 a$ and $b$ ). At $24 \mathrm{~h}$ after ischemia, an additional fragment of $105 \mathrm{kDa}$ was detectable in all samples, suggesting a further proteolytic cleavage of the substrate (Figure 3a).

Beclin-1 cleavage is dependent on calpain activity and NMDA receptor activation. To identify the temporal 

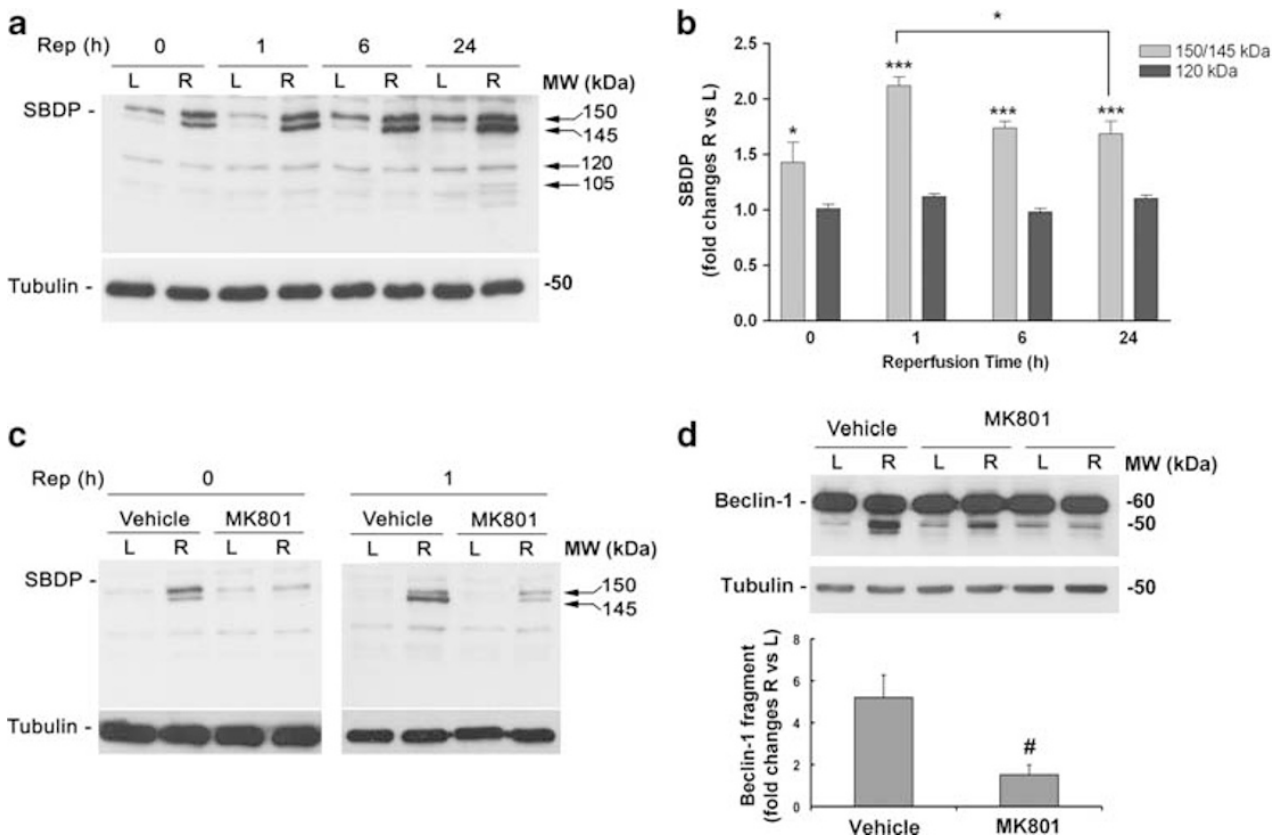

Figure 3 Blockade of NMDA-R by MK801 prevents calpain activation and Beclin-1 cleavage following retinal ischemia. (a) Immunoblotting showing the increase of calpain-specific 150/145-kDa SBPDs in the ischemic retina $(R)$ after 50 min of ischemia $(\operatorname{Rep}=0)$ and 1, 6 or $24 \mathrm{~h}$ of reperfusion. Note the lack of accumulation of the $120-k D a$ caspase-3 product. Histograms in (b) show the results of the densitometric analysis of the autoradiographic bands relative to 150/145- and 120-kDa SBDP normalized to the value of tubulin and compared with the left eye (L). (c) MK801 reduced the calpain activation induced by retina ischemia/reperfusion at the end of the ischemia (left panel) and after $1 \mathrm{~h}$ of reperfusion (right panel), as shown by the reduced intensity of the 150/145-kDa SBDP. A representative immunoblot from three independent experiments is shown. (d) Effect of MK801 treatment on Beclin-1 cleavage. Treatment with the NMDA antagonist significantly prevented the accumulation of the 50-kDa Beclin-1 fragment observed at $1 \mathrm{~h}$ of reperfusion. Histograms show the results of the densitometric analysis of Beclin-1 fragment immunoreactive band normalized to the value of loading control. Each value is the mean \pm S.E.M. of three experiments. In histogram $\mathbf{b}$, ${ }^{*} P<0.05$ versus $L$; ${ }^{* *} P<0.001$ versus $L$; and ${ }^{\#} P<0.05$ versus vehicle. MW, molecular weight; $R$, right ischemic eye; Rep, reperfusion time

sequence of events leading to Beclin-1 cleavage, we first investigated the consequences of an NMDA receptor block on calpain activation and Beclin-1 modulation.

Intravitreal administration of the use-dependent, noncompetitive NMDA receptor antagonist MK801 $(50 \mathrm{nmol}$, given $5 \mathrm{~min}$ before ischemia and at the beginning of the reperfusion) abolished calpain activation, as shown by the reduction of the $150 / 145-\mathrm{kDa}$ fragments (Figure $3 \mathrm{c}$ ). The same treatment also reduced the accumulation of the $50-\mathrm{kDa}$ Beclin-1 fragment at $1 \mathrm{~h}$ of reperfusion (Figure $3 \mathrm{~d}$ ).

To further correlate Beclin-1 cleavage to activation of calpains, the effect of the two calpain inhibitors MDL28170 (calpain inhibitor III) and SJA6017 was studied.

As expected, intravitreal injection of the calpain inhibitors $(1 \mathrm{mM}, 3 \mu \mathrm{l} / \mathrm{eye}$, given intravitreally $5 \mathrm{~min}$ before ischemia induction and at the beginning of reperfusion) blocked calpain activity at $1 \mathrm{~h}$ of reperfusion, as shown by the absence of the typical 150/145-kDa fragments (Figure 4). Pharmacological inhibition of calpains was paralleled by a significant reduction in the formation of the Beclin-1 fragment and by the increase of the full-length protein at $1 \mathrm{~h}$ of reperfusion (Figure 4).

Finally, knockdown of both $\mu$ - and m-calpain isoforms was performed by using a calpain small-subunit 1 small interfering RNA (CAPNS1 siRNA).

Effectiveness of silencing was checked by monitoring the appearance of the 150/145-kDa calpain-mediated SBDP in retinas subjected to ischemia and reperfused for $1 \mathrm{~h}$ (Figure 5a). A substantial reduction in $\alpha$-spectrin proteolysis was detected in the retinas of rats receiving the intravitreal injection of siRNA ( $10 \mu \mathrm{g} / \mathrm{eye}, 48 \mathrm{~h}$ before ischemia) when compared with the vehicle and the non-targeting scramble siRNA-treated animals (Figure 5a).

Silencing of CAPNS1 completely prevented the appearance of the $50-\mathrm{kDa}$ Beclin- 1 fragment typically observed in the ischemic retinas after $1 \mathrm{~h}$ of reperfusion (Figure $5 \mathrm{~b}$ ), thus confirming that, in our experimental setting, calpains were responsible for Beclin-1 cleavage.

Autophagy promotes RGC-5 survival. Depending on the context, autophagy has been shown to have either prosurvival or pro-death functions. To test the role of autophagy on RGCs survival, we induced autophagy in RGC- 5 cells by serum starvation ${ }^{18}$ and assayed cell viability.

Cells starved for $24 \mathrm{~h}$ showed a significant increase of Beclin-1 and LC3II levels (Figure 6a) and an altered intracellular localization of LC3, as revealed by immunofluorescence (Figure 6b). Cells grown in nutrient-rich medium (10\% serum; CTR) showed a diffused LC3 cytoplasmic distribution, whereas cells that have undergone serum deprivation (SD) showed intense dot-like LC3-positive structures, indicating the localization of LC3II on autophagic membranes and the increase of autophagosome formation (Figure 6b).

Serum starvation significantly reduced $\mathrm{RGC}-5$ cell viability (Figure 6c). To determine the effects of autophagy inhibition on cell survival, cells were treated with two autophagy 

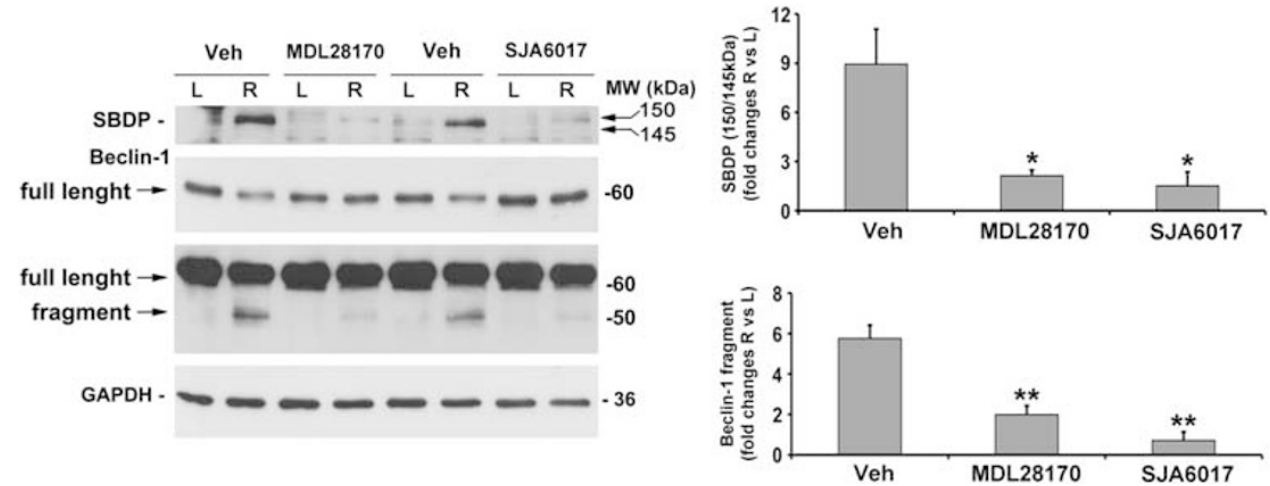

Figure 4 Beclin-1 cleavage depends on calpain activation. Representative western blotting showing the effect of calpain inhibitors MDL28179 and SJA6017 on calpain activation and Beclin-1 cleavage at $1 \mathrm{~h}$ of reperfusion. Calpain inhibition by intravitreal treatment with MDL28170 or SJA6017 completely prevented the accumulation of the $150 / 145-k D a$ SBDP, and significantly reduced Beclin-1 cleavage as shown by the reduction of the 50-kDa band corresponding to Beclin-1 fragment and the recovery of the 60-kDa full-length protein band. Control animals were subjected to intravitreal injection of the vehicle corresponding to the treatment (DMSO 10\%). Densitometric analyses of the autoradiographic bands, normalized to the value of the loading control, are reported in the histograms. Values are the mean \pm S.E.M. of three independent experiments. ${ }^{\star} P<0.05,{ }^{\star *} P<0.01$ versus vehicle. L, left eye; $M W$, molecular weight; $R$, right ischemic eye

a
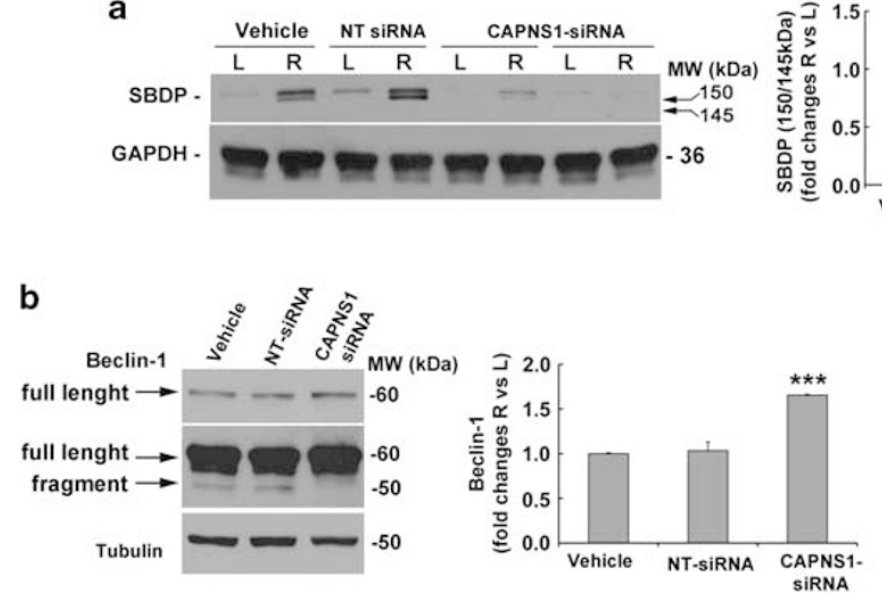

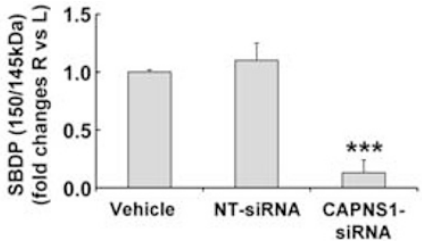

SIRNA

Figure 5 Beclin-1 proteolytic cleavage is abrogated by calpain knockdown. (a) Effectiveness of calpain small-subunit (CAPNS1) silencing. In vivo knockdown of calpains by intravitreal injection of a specific CAPNS-siRNA was verified by the abrogation of the calpain proteolytic activity on $\alpha$-spectrin in animals subjected to 50 min of ischemia, followed by $1 \mathrm{~h}$ of reperfusion. Western blotting analysis showed no changes of calpain proteolytic activity in the retinas from animals treated with the scramble non-targeting (NT) siRNA. Densitometric analysis of the $150 / 145-k D a$ SBDP bands showed a reduction by $87 \%$ in the CAPNS1 siRNA-treated animals (CAPNS1-siRNA $=0.13 \pm 0.11$ versus vehicle $=1 \pm 0.02$ ). (b) Beclin-1 cleavage was completely abrogated in the CAPNS1-silenced retinas subjected to ischemia, as shown by the absence of the $50-k D a$ proteolytic fragment and the recovery of the full-length protein observed at $1 \mathrm{~h}$ of reperfusion. Histograms show the densitometric analysis of the autoradiographic bands. Each value is the mean \pm S.E.M. of three independent experiments. ${ }^{* \star} P<0.001$ versus vehicle and NT siRNA. L, left eye; MW, molecular weight; $R$, right ischemic eye

inhibitors: bafilomycin A1 (BafA1), which increases intravesicular $\mathrm{pH}$ and therefore inhibits autophagy completion, ${ }^{19}$ and 3-methyladenine (3-MA), a nucleotide derivative that blocks class III PI3K activity ${ }^{20}$ upstream of LC3II formation.

Treatment of RGC-5 with BafA1 $\left(100 \mathrm{nM}^{18}\right)$ for $24 \mathrm{~h}$ strongly increased the accumulation of the lipidated form of LC3 and significantly reduced cell viability under starvation (46 versus $69 \%$ of serum-starved cells; $P<0.05$; Figures $6 \mathrm{~d}$ and e). A similar effect on cell survival was obtained when the cells were exposed to $3-\mathrm{MA}\left(10 \mathrm{mM}^{18}\right)$ during the $24 \mathrm{~h}$ of SD (16 versus $69 \%$ of serum-starved cells; $P<0.05$; Figure $6 e$ ).

Beclin-1 silencing reduces RGC-5 viability under starvation. To investigate the effects of Beclin-1 reduction on RGC survival, cells were transfected with rat Beclin-1-
siRNA $48 \mathrm{~h}$ before SD. Gene silencing reduced Beclin-1 expression by $95 \%$ compared with starved scrambletransfected cells as shown by western blotting analysis (Figure 7a) and led to a reduction of LC3II formation under starvation (Figure 7a) similar to the pattern observed in vivo (Figure 2). Beclin-1 silencing significantly worsened the reduction of RGC-5 viability, as assessed by 3-(4,5dimethyl-2-thiazolyl)-2,5-diphenyl-2H-tetrazolium bromide (MTT) assay, after $24 \mathrm{~h}$ of starvation (36 versus $67 \%$ of serum-starved cells; $P<0.05$; Figure 7b).

\section{Discussion}

Depending on the circumstances, autophagy can have both beneficial and detrimental effects on neurons. Blocking of 

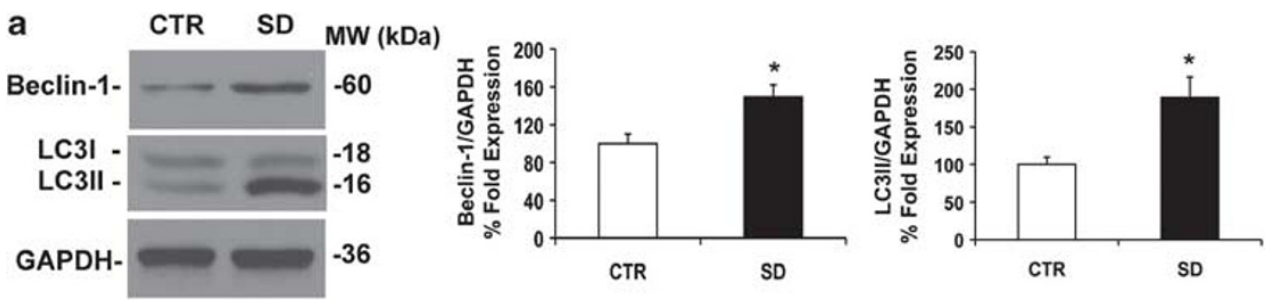

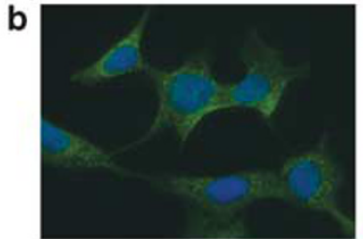

CONTROL

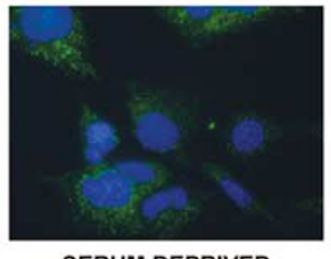

SERUM DEPRIVED
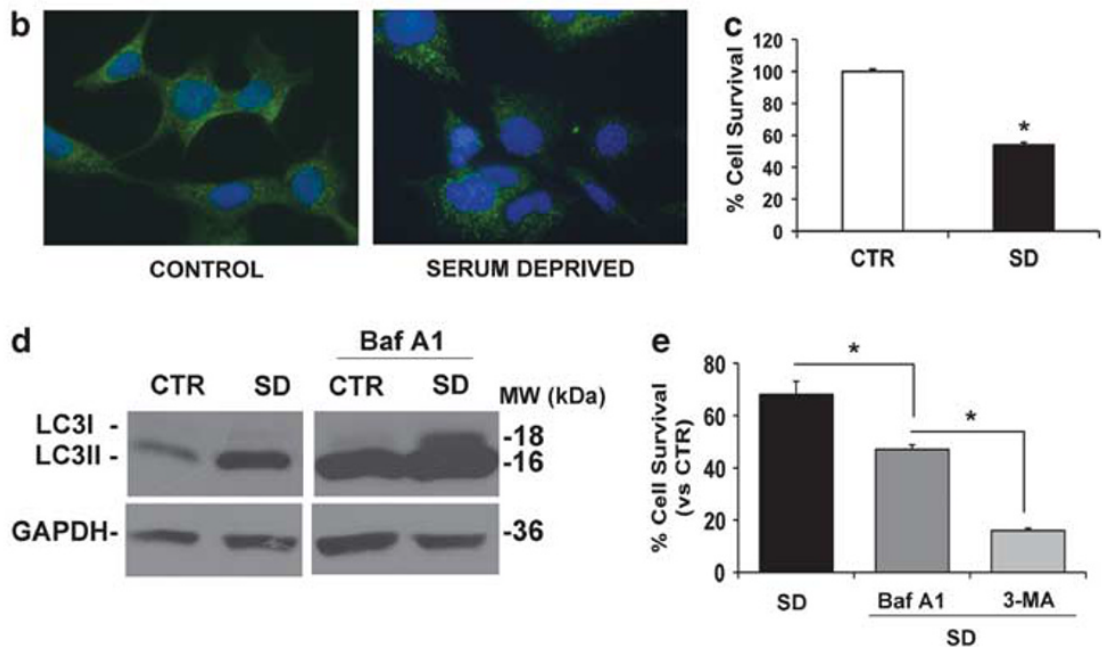

Figure 6 SD induces autophagy in RGC-5 cells. (a) Effect of SD on Beclin-1 and LC3 expression. Representative western blot showing the expression of Beclin-1 and LC3I and II in RGC-5 cell control (10\% FBS; CTR) and starved (0\% FBS; SD) for $24 \mathrm{~h}$. Histograms show densitometric analysis. Data were normalized for GAPDH and expressed as percentage of control values, $n=5,{ }^{*} P<0.05$. (b) Immunocytochemistry for LC3 in RGC-5 cells in presence (control) or absence of serum (serum deprived). After $24 \mathrm{~h}$ of SD, LC3 immunoreactivity shows a punctuate pattern due to the localization of LC3Il in the autophagosome membrane. (c) SD reduces RGC-5 cell survival. Cell viability was measured by MTT assay (CTR $=100 \pm 1.5$; S.D. $=54 \pm 1.5 ; n=5$ ). (d) Effect of BafA1 treatment on LC3 expression. Western blot, representative of three independent experiments, shows the accumulation of LC3I and II in RGC-5 treated with BafA1 in presence or absence of serum for $24 \mathrm{~h}$. (e) Effect of treatment with autophagy inhibitors on cell survival of starved RGC-5. RGC-5 cells were treated with BafA1 ( $100 \mathrm{nM}$ ) and 3-MA (10 mM) for $24 \mathrm{~h}$ under SD and viability evaluated by MTT assay ( $n=3)$. Each bar represents the mean \pm S.E.M. ${ }^{*} P<0.05$

autophagy causes neurodegeneration, ${ }^{3,4}$ and alterations of the autophagy pathway have been observed in chronic neurodegenerative conditions, including $A D,{ }^{21} \mathrm{PD}^{22}$ and Huntington's disease. ${ }^{23}$ Autophagy is also activated in neuronal cells exposed to hypoxic, ${ }^{24}$ excitotoxic ${ }^{25}$ or ischemic injuries. ${ }^{26}$

Retinal ischemia is a common clinical condition and a relevant cause of visual impairment and blindness. Ocular ischemia induced by transient elevation of IOP in rat is a common animal model used to investigate the mechanisms underlying retinal neuronal death. With this method, a global ischemia is produced by the obstruction of both retinal and uveal circulation, leading to pathological features similar to those observed after central retinal artery occlusion and acute angle-closure glaucoma. ${ }^{8} \mathrm{We}$ investigated the participation of autophagy in the neurodegenerative processes accompanying ischemia in the retina following IOP and observed a decrease in LC3II, the autophagosomal associated form of LC3. This was accompanied by Beclin-1 reduction during the reperfusion phase and was indicative of a general deregulation of the retinal autophagic machinery following ischemia/ reperfusion. Although Beclin-1 appeared to be expressed throughout all retinal layers, higher immunoreactivity was detected in the RGCs, suggesting a prevalence of the autophagic process in this neuronal type.
In our model, Beclin-1 reduction was associated with the appearance of a $50-\mathrm{kDa}$ fragment in the ischemic retinas during the first hour of reperfusion, suggesting the occurrence of a proteolytic cleavage. Caspases 3, 7 and 8 have recently been reported to cleave Beclin-1, producing two proteolytic fragments of 35 and/or $37 \mathrm{kDa}^{27,28}$ However, the late time of caspase activation following ischemia in the retina (12 $\mathrm{h}$ after reperfusion ${ }^{29}$ ), as well as the different molecular weights of the fragments seemed to exclude the involvement of this cascade in this cleavage. Therefore, we investigated the role of the major alternative degradation pathway of calpains, whose activation has been reported after ocular hypertension in rat ${ }^{30}$ as well as in primate retinas during hypoxia. ${ }^{31}$ Calpain inhibition by MDL28170 and SJA6017, as well as calpains silencing by siRNA prevented the formation of the fragment, thus indicating the contribution of these enzymes to Beclin-1 cleavage in our model. Calpain-mediated cleavage of Beclin-1 was previously reported in recombinant assay in vitro ${ }^{32}$ and in cultured hepatocytes exposed to anoxia/reoxygenation. ${ }^{18,33}$ However, to the best of our knowledge, our data represent the first evidence of Beclin-1 cleavage in neuronal tissue and the first report of Beclin-1 as a substrate of calpains in vivo.

Calpains are calcium-dependent enzymes activated by intracellular $\mathrm{Ca}^{2+}$ increase consequent to glutamate receptors overactivation and associated with excitotoxicity. ${ }^{16}$ 
a
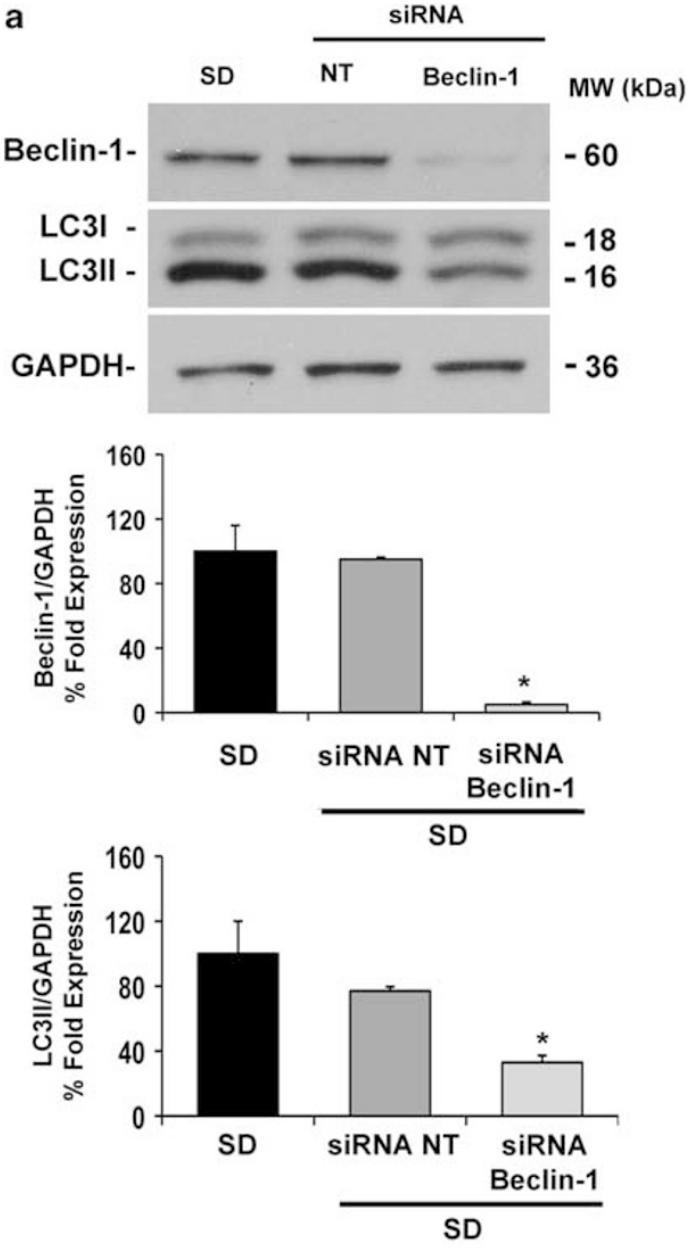

b

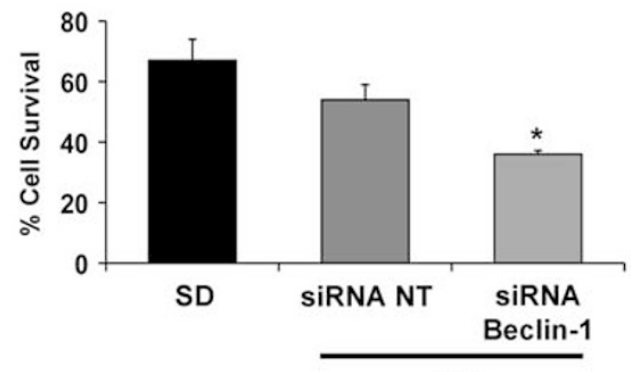

SD

Figure 7 Beclin-1 silencing reduces RGC-5 viability following SD. (a) Effectiveness of Beclin-1 silencing and effect on LC3. Transfection of specific Beclin-1-siRNA or scramble non-targeting (NT) sequence at final concentration of $25 \mathrm{nM}$ was performed, $48 \mathrm{~h}$ before SD, in 96-well plate with Lipofectamine 2000 according to the manufacturer's instructions. In Beclin-1-siRNA-transfected cells, expression of the protein was reduced by $95 \%$ and was paralleled by LC3II reduction. Histograms show the densitometric analysis of autoradiographic bands. Data were normalized for GAPDH and expressed as percentage of control values $(n=3)$. (b) Effect of Beclin-1 knockdown on survival of serum-deprived RGC-5 cells. Each bar represents the mean \pm S.E.M. $(n=3)$ of cell viability, as assessed by the MTT assay. ${ }^{*} P<0.05$ versus NT siRNA and SD (SD, $0 \%$ FBS). MW, molecular weight

The latter contributes to a variety of acute and chronic neurodegenerative diseases and has a key role also in RGC death associated with retinal ischemia. ${ }^{10}$ However, the relation between autophagy and excitotoxic cell death is still controversial. NMDA treatment of organotypic hippocampal slices, as well as striatal injection of kainic acid, is associated with induction of autophagy. In these same models, inhibition of autophagosomal formation prevented neuronal death, ${ }^{25,34}$ linking excitotoxicity to induction of autophagy via a common $\mathrm{Ca}^{2+}$-mediated pathway. In contrast, recent studies identified inhibitors of intracellular $\mathrm{Ca}^{2+}$ currents as activators of autophagy ${ }^{35,36} \mathrm{In}$ agreement with the latter observation, our data would support $\mathrm{a} \mathrm{Ca}^{2+}$-mediated inhibition of autophagy via the calpain-mediated Beclin-1 cleavage and provide a possible link between excitotoxicity and the impaired autophagy often observed in neurodegenerative diseases.

NMDA receptor antagonists and calpain inhibitors have been shown to reduce neurodegeneration triggered by ischemia in the retina. ${ }^{11,30}$ Thus, the calpain inhibitor SJA6017 reduced cell loss in the GCL after retinal ischemia-reperfusion; ${ }^{30}$ furthermore, neuroprotection by calpain inhibition has been also reported in an acute ocular hypertension/ischemia model ${ }^{37}$ and following NMDA-induced retinal damage. ${ }^{38}$ Our data suggest that prevention of Beclin-1 cleavage and autophagy deregulation might be implicated in the neuroprotection afforded by NMDA receptor antagonists and calpain inhibitors in retinal ischemia-reperfusion injury.

To date, very little is known on the role of the autophagic pathway in RGC death. Activation of autophagy was observed in RGC following optic nerve transection, and a protective role was suggested for this process in RGC-5 cells under starvation. ${ }^{18}$ A persistent accumulation of autophagosomes, concurring to the injury-induced axonal degradation and secondary to the lesion-induced calcium influx, was reported in the optic nerve following optic nerve crush. ${ }^{39}$

In our study, Beclin-1 knockdown in starved RGC-5 prevented LC3II formation, thereby the induction of autophagy, and increased the cell death rate. This result, in accordance with previous work, ${ }^{18}$ points toward a neuroprotective role of autophagy in RGC exposed to detrimental stimuli.

In summary, our findings provide evidence of the autophagy impairment in the ischemic retina, support a neuroprotective role of autophagy in RGCs and suggest that excitotoxicity negatively regulates autophagy through the calpain-mediated cleavage of Beclin- 1 .

In recent years, the connection between autophagy and neurodegeneration has been strengthened by several studies highlighting the vulnerability of neurons to stress-related signals that impair the autophagic process. Our study suggests that alterations of the autophagic pathway might be an important aspect in retinal pathologies associated with ischemic events and, therefore, a potential target for new and supporting therapeutic interventions.

\section{Materials and Methods}

Retinal ischemia injury. Male Wistar rats (280-330 g) were purchased from Charles River (Lecco, Italy). Animals were housed with a 12-h light-dark cycle with ad libitum access to food and water. Animal care and experiments were carried out in accordance with the guidelines of the Italian Ministry of Health for Animal care (DM 116/1992).

Retinal ischemia was induced in adult rats by acutely increasing the IOP according to the method previously reported. ${ }^{15}$ 
Animals were deeply anesthetized by intraperitoneal injection of urethane $(1.5 \mathrm{~g} / \mathrm{kg})$ and laid on a heating pad to maintain the body temperature at $37^{\circ} \mathrm{C}$. Topical anesthesia was induced by $0.4 \%$ oxibuprocain eye drops (Novesina, Novartis, Varese, Italy). A 27-gauge infusion needle, connected to a $500-\mathrm{ml}$ bottle of sterile saline, was inserted in the anterior chamber of the right eye, and the saline container was elevated to produce an IOP of $120 \mathrm{~mm} \mathrm{Hg}$ for $50 \mathrm{~min}$. Retinal ischemia was confirmed by whitening of the fundus. For each animal, the left eye was used as non-ischemic control.

Body temperature was monitored before and after ischemia, and animals with a value lower than $35.5^{\circ} \mathrm{C}$ were excluded. Animals were killed by cervical dislocation at the end of the ischemia or at $1,6,24 \mathrm{~h}$ or 7 days after ischemia. Eyes were removed, retinas quickly dissected, snap frozen in liquid nitrogen and stored at $-80^{\circ} \mathrm{C}$ until use. Time points were chosen based on our previous study. ${ }^{15}$

Cell culture and treatments. The rat RGC line (RGC-5), established by transforming rat postnatal day 1 retinal cells with the $2 \Psi$ E1A virus, ${ }^{40}$ was purchased from the American Type Culture Collection (Manassas, VA, USA). Cells were cultured in high-glucose Dulbecco's Modified Eagle's Medium with L-glutamine (Invitrogen, Carlsbad, CA, USA), supplemented with 10\% fetal bovine serum (FBS; Invitrogen) and were grown at $37^{\circ} \mathrm{C}$ in a humidified atmosphere with $5 \% \mathrm{CO}_{2}$. Depending on the experiment performed, cells were seeded in 6- or 96-well plates, in $10 \mathrm{~cm}$ petri dishes or coverslip and grown for $24 \mathrm{~h}$ before treatments.

Cells were subjected to starvation in serum-free media for $24 \mathrm{~h}$. Where indicated, Bafilomycin A1 (BafA1; $100 \mathrm{nM}$; Sigma-Aldrich, Milan, Italy) or 3-MA (10 mM; Sigma-Aldrich) were added to the medium and maintained during the starvation period. Control cells were treated with the corresponding amount of vehicles.

Intravitreal administrations. The NMDA antagonist MK801 (SigmaAldrich) was dissolved in sterile phosphate-buffered saline (PBS) A stock solution of the calpain inhibitors MDL28170 or SJA6017 (Calbiochem, La Jolla, CA, USA) was prepared in $100 \%$ dimethylsulfoxide (DMSO) at the concentration of 10 and $20 \mathrm{mM}$, respectively. The stocks were subsequently diluted to the final concentration using sterile PBS.

Intravitreal injection was performed by puncturing the eye with a 23-gauge needle at the cornea-sclera junction and the drugs administered with a 5- $\mu$ l Hamilton syringe (Bonaduz, GR, Switzerland). MK801 (50 nmol/5 $\mu / /$ eye $){ }^{15}$ MDL28170 (0.5-1.0 mM/3 $\mu / /$ eye), SJA6017 $(0.5-1.0 \mathrm{mM} / 3 \mu \mathrm{l} / \mathrm{eye})$ or control solution (2.5-5\% DMSO in PBS/3 $\mu / /$ eye) was administered $5 \mathrm{~min}$ before and at the end of the ischemia. The duration of the injection was 3 min in all instances. At the indicated time points, animals were killed, and subjects with visible lens damage or vitreous hemorrhage were excluded from the study.

Knockdown of CAPNS1 and Beclin-1 expression by siRNA. An siRNA specific for rat Beclin-1 (coiled-coil, myosin-like Bcl-2-interacting protein, NM053739; ON-TARGET plus SMARTpool L-099237-01) or rat CAPNS1 (NM017118; ON-TARGET plus SMARTpool L-082111-01) was obtained from Dharmacon RNAi Technologies (Chicago, IL, USA). The siRNA was reconstituted in sterile RNase-free water at a final concentration of $100 \mu \mathrm{M}$. In all, $10 \mu \mathrm{g}$ of the siRNA was delivered intravitreally $72 \mathrm{~h}$ before inducing ischemia using a $10 \mu \mathrm{l}$ Hamilton syringe. Scramble control siRNA (ON-TARGET plus Non-Targeting Pool D-001819-10) was injected to exclude non-specific effect of the silencing.

For in vitro studies, RGC- 5 was transfected, $24 \mathrm{~h}$ before starvation, with specific siRNA at a final concentration of $25 \mathrm{nM}$ using Lipofectamine 2000 transfection reagent (Invitrogen) according to the manufacturer's instruction.

Immunoblot analysis. Retinas or RGC-5 cells were lysed in ice-cold RIPA buffer (50 mM Tris-HCl (pH 8), $150 \mathrm{mM} \mathrm{NaCl}, 1 \mathrm{mM}$ EDTA, 0.1\% SDS, $1 \%$ IGEPAL and $0.5 \%$ sodium deoxicholate) containing protease (code P8349; Sigma-Aldrich) and phosphatase inhibitor cocktails (code 524625; Calbiochem). Lysates were centrifuged for $15 \mathrm{~min}$ at $10000 \times \mathrm{g}$ at $4^{\circ} \mathrm{C}$, and supernatants were assayed for protein content by the Bio-Rad DC Protein Assay Kit (Bio-Rad laboratories, Milan, Italy).

For western blot analysis, equal amount of total proteins was separated by SDSpolyacrylamide gel electrophoresis ( $8 \%$ for $\alpha$-spectrin; $12 \%$ for Beclin-1, Atg 4 , Atg7 and p62; and 15\% for LC3) and transferred onto PVDF membranes (Immobilon-P, Sigma-Aldrich). The membranes were blocked with $5 \%$ non-fat milk in Tris-buffered saline containing $0.05 \%$ Tween 20 for $1 \mathrm{~h}$ at room temperature (RT). Primary antibodies were incubated overnight at $4^{\circ} \mathrm{C}$, followed by a horseradish peroxidase-conjugated secondary antibody for $1 \mathrm{~h}$ at RT. Protein bands were visualized with the ECL Western Blotting Detection kit (ECL, Amersham Biosciences, GE Healthcare, Milan, Italy), and the chemiluminescence signal detected using X-ray films (Hyperfilm ECL, Amersham Biosciences). Exposure time to obtain the optimal density of the images varied from $5 \mathrm{~s}$ to $20 \mathrm{~min}$. Autoradiographic films were scanned, digitalized at $300 \mathrm{dpi}$ and band quantification was performed using ImageJ software (NIH, Bethesda, MD, USA).

The following primary antibodies and dilutions were used: anti-Beclin-1 (code PD017) 1: 4000, anti-p62/SQSTM1 1: 1000, anti-LC3 (code PD036) 1: 2000 (MBL International Corporation, Nagoya, Japan); rabbit monoclonal anti-Beclin-1 1:2000, anti-Atg4 1:1000, anti-Atg7 1:1000 (Cell Signaling Technology, Beverly, MA, USA); anti-spectrin (non-erythroid) monoclonal antibody (MAB 1622) 1:3000 (Chemicon International Inc., Temecula, CA, USA); anti-actin 1: 1000 (clone AC-40, Sigma-Aldrich); anti- $\beta$-tubulin 1:20000 (clone B-5-1-2, Sigma-Aldrich); and antiGAPDH 1:30000 (Applied Biosystems, Carlsbad, CA, USA). Species-specific horseradish peroxidase-conjugated goat IgG (Pierce Biotechnology, Rockford, IL, USA) were used as secondary antibodies.

Cell viability assay. Cell viability, evaluated as mitochondrial activity, was quantified by measuring dehydrogenase activity retained in the cells by using the MTT (Sigma-Aldrich) assay. The assay is based on the ability of living cells to reduce MTT into insoluble formazan. Briefly, cells (1500 cell per well) were seeded in 96-well plates and subjected to the appropriate treatment. After $24 \mathrm{~h}$, cells were incubated with $0.5 \mathrm{mg} / \mathrm{ml} \mathrm{MTT} \mathrm{(100 \mu l} \mathrm{per} \mathrm{well)} \mathrm{for} 2 \mathrm{~h}$ in a humidified $5 \% \mathrm{CO}_{2}$ incubator at $37^{\circ} \mathrm{C}$. Medium was then removed, and $100 \mu$ of DMSO (Carlo Erba, Milan, Italy) was added to solubilize the formazan product. Absorbance was read at $540 / 690 \mathrm{~nm}$ by using an ELISA reader (Labsystems Multiskan, Helsinki, Finland). Data were expressed as cell survival percentage versus control cultures (maintained in $10 \%$ FBS) set to $100 \%$.

Immunohistochemistry and immunocytochemistry. Immediately after the killing, eyes were enucleated and fixed in $2 \%$ paraformaldehyde (PFA) at $4{ }^{\circ} \mathrm{C}$ for $10 \mathrm{~min}$; the anterior segment was removed, the posterior was fixed in $4 \%$ PFA for $60 \mathrm{~min}$ and cryopreserved in $30 \%$ sucrose overnight. Specimens were frozen in Optimal Cutting Temperature compound (Tissue-Tek, Sakura Finetek Europe, Alphen aan den Rijn, The Netherlands), and 10- $\mu \mathrm{m}$ cryostat sections were cut, mounted onto Superfrost ultra plus glass slide (Menzel-Gläser, Braunschweig, Germany) and stored at $-80^{\circ} \mathrm{C}$ until used.

Retinal sections were thawed, air dried and washed in 0.1-M PBS (pH 7.4). Sections were permeabilized with $0.3 \%$ Triton for $30 \mathrm{~min}$ and blocked with $10 \%$ donkey serum (Sigma-Aldrich) at RT for $1 \mathrm{~h}$. The slides were then incubated with rabbit anti-Beclin-1 (1:50, MBL International Corporation) and mouse antiTUJ1 ( $\beta$ III tubulin; 1:500; BabCo, Richmond, CA USA), to identify adult RGCs (Cui et al., 2003; Huang et al., 2008), or with mouse anti-GFAP (1:800; MAB360; Chemicon/Millipore, Billerica, MA, USA) to identify Müller cells. Finally, sections were incubated with anti-rabbit Alexa Fluor $488(1: 300)$ and/or anti-mouse Alexa Fluor 594 (1:500; Molecular Probes, Eugene, OR, USA) at RT for $1 \mathrm{~h}$ and mounted with Vectashield mounting media with DAPI (Vector Laboratories, Burlingame, CA, USA).

For immunocytochemistry, RGC- 5 cells were plated on coverslips, fixed with $4 \%$ PFA for $20 \mathrm{~min}$ at RT, permeabilized with Triton $0.3 \%$ for $5 \mathrm{~min}$ and blocked with $10 \%$ donkey serum (Sigma-Aldrich) in PBS for $30 \mathrm{~min}$. LC3 protein was detected by incubating with anti-LC3 antibody (1:200; MBL International Corporation) in 5\% blocking buffer for $1 \mathrm{~h}$ at RT, followed by Alexa Fluor 488 (1:500; Molecular Probes) in $5 \%$ blocking buffer for $1 \mathrm{~h}$ at RT. Coverslips were mounted with Vectashield solution containing DAPI (Vector Laboratories).

Image acquisition was performed using a deconvolution microscope (Leica EL6000 microsystem, Milan, Italy).

Statistical analysis. Data are given as mean \pm S.E.M. of three to six independent experiments and statistically evaluated for difference by Student's $t$-test or by one-way analysis of variance, followed by Newman-Keuls or TukeyKramer test for multiple comparisons. A value of $P \leq 0.05$ was considered statistically significant.

\section{Conflict of interest}

The authors declare no conflict of interest. 
Acknowledgements. We gratefully acknowledge the partial financial support from the University of Calabria (ex quota $60 \%$ ). We also thank Mr Guido Fico for skillful technical support.

1. Klionsky DJ. The molecular machinery of autophagy: unanswered questions. J Cell Sci 2005; 118: 7-18.

2. Levine B, Kroemer G. Autophagy in the pathogenesis of disease. Cell 2008; 132: 27-42.

3. Hara T, Nakamura K, Matsui M, Yamamoto A, Nakahara Y, Suzuki-Migishima R et al. Suppression of basal autophagy in neural cells causes neurodegenerative disease in mice. Nature 2006; 441: 885-889.

4. Komatsu M, Wang QJ, Holstein GR, Friedrich Jr VL, Iwata J, Kominami E et al. Essential role for autophagy protein Atg7 in the maintenance of axonal homeostasis and the prevention of axonal degeneration. Proc Natl Acad Sci USA 2007; 104: 14489-14494.

5. Fimia GM, Stoykova A, Romagnoli A, Giunta L, Di Bartolomeo S, Nardacci R et al. Ambra1 regulates autophagy and development of the nervous system. Nature 2007; 447 $1121-1125$.

6. Jaeger PA, Wyss-Coray T. All-you-can-eat: autophagy in neurodegeneration and neuroprotection. Mol Neurodegener 2009; 4: 16.

7. Wong $\mathrm{E}$, Cuervo AM. Autophagy gone awry in neurodegenerative diseases. Nat Neurosc 2010; 13: 805-811.

8. Osborne NN, Casson RJ, Wood JP, Chidlow G, Graham M, Melena J. Retinal ischemia: mechanisms of damage and potential therapeutic strategies. Prog Retin Eye Res 2004; 23 91-147.

9. Kaur C, Foulds WS, Ling EA. Hypoxia-ischemia and retinal ganglion cell damage. Clin Ophthalmol 2008; 2: 879-889.

10. Russo R, Rotiroti D, Tassorelli C, Nucci C, Bagetta G, Bucci MG et al. Identification of nove pharmacological targets to minimize excitotoxic retinal damage. Int Rev Neurobiol 2009; 85: 407-423.

11. Nucci C, Tartaglione R, Rombola L, Morrone LA, Fazzi E, Bagetta G. Neurochemical evidence to implicate elevated glutamate in the mechanisms of high intraocular pressure (IOP)-induced retinal ganglion cell death in rat. Neurotoxicology 2005; 26: 935-941.

12. Fujita $R$, Ueda $M$, Fujiwara $K$, Ueda $H$. Prothymosin-alpha plays a defensive role in retinal ischemia through necrosis and apoptosis inhibition. Cell Death Differ 2009; 16: 349-358.

13. Sinha S, Levine B. The autophagy effector Beclin 1: a novel BH3-only protein. Oncogene 2008; 27(Suppl 1): S137-S148.

14. Kabeya Y, Mizushima N, Ueno T, Yamamoto A, Kirisako T, Noda T et al. LC3, a mammalian homologue of yeast Apg8p, is localized in autophagosome membranes after processing. EMBO J 2000; 19: 5720-5728.

15. Russo R, Cavaliere F, Berliocchi L, Nucci C, Gliozzi M, Mazzei C et al. Modulation of pro-survival and death-associated pathways under retinal ischemia/reperfusion: effects of NMDA receptor blockade. J Neurochem 2008; 107: 1347-1357.

16. Lynch DR, Guttmann RP. Excitotoxicity: perspectives based on N-methyl-D-aspartate receptor subtypes. J Pharmacol Exp Ther 2002; 300: 717-723.

17. Wang KK. Calpain and caspase: can you tell the difference? Trends Neurosci 2000; 23 20-26.

18. Kim SH, Munemasa Y, Kwong JM, Ahn JH, Mareninov S, Gordon LK et al. Activation of autophagy in retinal ganglion cells. J Neurosci Res 2008; 86: 2943-2951.

19. Yamamoto A, Tagawa Y, Yoshimori T, Moriyama Y, Masaki R, Tashiro Y. Bafilomycin A1 prevents maturation of autophagic vacuoles by inhibiting fusion between autophagosomes and lysosomes in rat hepatoma cell line, H-4-II-E cells. Cell Struct Funct 1998; 23: 33-42.

20. Petiot A, Ogier-Denis E, Blommaart EF, Meijer AJ, Codogno P. Distinct classes of phosphatidylinositol $3^{\prime}$-kinases are involved in signaling pathways that control macroautophagy in HT-29 cells. J Biol Chem 2000; 275: 992-998.

21. Nixon RA, Wegiel J, Kumar A, Yu WH, Peterhoff C, Cataldo A et al. Extensive involvement of autophagy in Alzheimer disease: an immuno-electron microscopy study. J Neuropathol Exp Neurol 2005; 64: 113-122.

22. Pan T, Kondo S, Le W, Jankovic J. The role of autophagy-lysosome pathway in neurodegeneration associated with Parkinson's disease. Brain 2008; 131: 1969-1978.

23. Ravikumar B, Vacher C, Berger Z, Davies JE, Luo S, Oroz LG et al. Inhibition of mTOR induces autophagy and reduces toxicity of polyglutamine expansions in fly and mouse models of Huntington disease. Nat Genet 2004; 36: 585-595.
24. Zhu C, Wang X, Xu F, Bahr BA, Shibata M, Uchiyama $Y$ et al. The influence of age on apoptotic and other mechanisms of cell death after cerebral hypoxia-ischemia. Cell Death Differ 2005; 12: 162-176.

25. Wang Y, Han R, Liang ZQ, Wu JC, Zhang XD, Gu ZL et al. An autophagic mechanism is involved in apoptotic death of rat striatal neurons induced by the non-N-methyl-D-aspartate receptor agonist kainic acid. Autophagy 2008; 4: 214-226.

26. Adhami F, Liao G, Morozov YM, Schloemer A, Schmithorst VJ, Lorenz JN et al. Cerebral ischemia-hypoxia induces intravascular coagulation and autophagy. Am J Pathol 2006; 169: 566-583.

27. Cho DH, Jo YK, Hwang JJ, Lee YM, Roh SA, Kim JC. Caspase-mediated cleavage of ATG6/Beclin-1 links apoptosis to autophagy in HeLa cells. Cancer Lett 2009; 274: 95-100.

28. Luo S, Rubinsztein DC. Apoptosis blocks Beclin 1-dependent autophagosome synthesis: an effect rescued by Bcl-xL. Cell Death Differ 2010; 17: 268-277.

29. Zhang B, Safa R, Rusciano D, Osborne NN. Epigallocatechin gallate, an active ingredien from green tea, attenuates damaging influences to the retina caused by ischemia/ reperfusion. Brain Res 2007; 1159: 40-53.

30. Sakamoto YR, Nakajima TR, Fukiage CR, Sakai OR, Yoshida YR, Azuma MR et al. Involvement of calpain isoforms in ischemia-reperfusion injury in rat retina. Curr Eye Res 2000; 21: 571-580.

31. Nakajima E, David LL, Bystrom C, Shearer TR, Azuma M. Calpain-specific proteolysis in primate retina: contribution of calpains in cell death. Invest Ophthalmol Vis Sci 2006; 47: 5469-5475.

32. Wirawan E, Vande Walle L, Kersse K, Cornelis S, Claerhout S, Vanoverberghe I et al. Caspase-mediated cleavage of Beclin-1 inactivates Beclin-1-induced autophagy and enhances apoptosis by promoting the release of proapoptotic factors from mitochondria. Cell Death Dis 2010; 1: e18.

33. Kim JS, Nitta T, Mohuczy D, O'Malley KA, Moldawer LL, Dunn Jr WA et al. Impaired autophagy: a mechanism of mitochondrial dysfunction in anoxic rat hepatocytes. Hepatology 2008; 47: 1725-1736.

34. Borsello T, Croquelois K, Hornung JP, Clarke PG. N-methyl-d-aspartate-triggered neuronal death in organotypic hippocampal cultures is endocytic, autophagic and mediated by the c-Jun N-terminal kinase pathway. Eur J Neurosci 2003; 18: 473-485.

35. Zhang L, Yu J, Pan H, Hu P, Hao Y, Cai W et al. Small molecule regulators of autophagy identified by an image-based high-throughput screen. Proc Natl Acad Sci USA 2007; 104: 19023-19028.

36. Williams A, Sarkar S, Cuddon P, Ttofi EK, Saiki S, Siddiqi FH et al. Novel targets for Huntington's disease in an mTOR-independent autophagy pathway. Nat Chem Biol 2008; 4: $295-305$

37. Oka T, Walkup RD, Tamada Y, Nakajima E, Tochigi A, Shearer TR et al. Amelioration of retinal degeneration and proteolysis in acute ocular hypertensive rats by calpain inhibitor ((1S)-1-((((1S)-1-benzyl-3-cyclopropylamino-2,3-di-oxopropyl)amino)carbony I)-3methylbutyl)carbamic acid 5-methoxy-3-oxapentyl ester. Neuroscience 2006; 141 2139-2145.

38. Shimazawa M, Suemori S, Inokuchi $Y$, Matsunaga N, Nakajima $Y$, Oka $T$ et al. A novel calpain inhibitor, ((1S)-1-((((1S)-1-Benzyl-3-cyclopropylamino-2,3-di-oxopropyl)amino) carbony I)-3-methylbutyl)carbamic acid 5-methoxy-3-oxapentyl ester (SNJ-1945), reduces murine retinal cell death in vitro and in vivo. J Pharmacol Exp Ther 2010; 332 380-387.

39. Knoferle J, Koch JC, Ostendorf T, Michel U, Planchamp V, Vutova P et al. Mechanisms of acute axonal degeneration in the optic nerve in vivo. Proc Natl Acad Sci USA 2010; 107: 6064-6069.

40. Krishnamoorthy RR, Agarwal P, Prasanna G, Vopat K, Lambert W, Sheedlo HJ et al. Characterization of a transformed rat retinal ganglion cell line. Brain Res Mol Brain Res 2001; 86: 1-12.

Cell Death and Disease is an open-access journal published by Nature Publishing Group. This work is licensed under the Creative Commons Attribution-Noncommercial-No Derivative Works 3.0 Unported License. To view a copy of this license, visit http://creativecommons.org/licenses/by-nc-nd/3.0/ 